\title{
The Eastern Townships Contemplated as a British Stronghold
}

\section{Maurice O’Bready}

Volume 15, numéro 2, septembre 1961

URI : https://id.erudit.org/iderudit/302113ar

DOI : https://doi.org/10.7202/302113ar

Aller au sommaire du numéro

Éditeur(s)

Institut d'histoire de l'Amérique française

ISSN

0035-2357 (imprimé)

1492-1383 (numérique)

Découvrir la revue

Citer cet article

O’Bready, M. (1961). The Eastern Townships Contemplated as a British

Stronghold. Revue d'histoire de l'Amérique française, 15(2), 230-255.

https://doi.org/10.7202/302113ar d'utilisation que vous pouvez consulter en ligne.

https://apropos.erudit.org/fr/usagers/politique-dutilisation/ 


\section{THE EASTERN TOWNSHIPS CONTEMPLATED AS A BRITISH STRONGHOLD}

\section{Préliminaires}

Pour donner une ambiance à notre sujet et faire mieux apercevoir le problème qu'il implique, nous retournons 200 ans en arrière en rappelant quelques événements déjà connus de nos lecteurs.

En 1763, après la victoire de Wolfe sur Montcalm, la Nouvelle-France passe à l'Angleterre. Les Français du Canada, qui depuis 150 ans ont échelonné leurs seigneuries le long du SaintLaurent, de la Chaudière et du Richelieu, s'accommodent tant bien que mal de leur nouvelle condition de conquis; surtout après 1774, alors que l'Acte de Québec adoucit leur sort en élargissant leur droit de citoyenneté. Ils se nombrent alors à environ 75,000, quand le vainqueur ne compte pas encore 200 de ses sujets dans la colonie.

Cette année-là, 1774, au delà de la ligne du 45e, les treize colonies de la Nouvelle-Angleterre, impatientées de la tracassière gouverne de la mère-patrie, se révoltent contre elle. Le conflit durera jusqu'en 1783, et mettra aux prises l'armée des Républicains, désireux de se libérer du joug britannique, et l'armée des Loyalistes (ou Royalistes), déterminés à rester fidèles ou «loyaux» au roi d'Angleterre. En histoire, on désigne du nom de Guerre d'Indépendance ce duel national, guerre civile qui opposa des compatriotes, et parfois des frères d'une même famille. Les Républicains gagnèrent la partie. Ils obtinrent de se séparer de l'Angleterre et formèrent, à même leurs treize colonies, la fédération des Etats-Unis d'Amérique.

On comprendra que les Loyalistes, défaits, détestés, molestés dans leurs biens comme dans leurs personnes, n'aient nourri dès lors qu'une seule idée: fuir les nouveaux Etats-Unis pour une terre plus hospitalière. Les uns regagnèrent l'Angleterre; 
d'autres (environ 30,000), montèrent dans les Maritimes, où ils firent souche; 30,000 aussi traversèrent le lac Ontario pour inaugurer la colonisation de townships autour de la Baie de Quinté (auj. Kingston), et leur nombre, comme leur langue et leur mentalité, leur mérita en 1791 un parlement distinct, celui du Haut-Canada; puis au bas mot 15,000 Loyalistes, empruntant le lac Champlain, se massèrent à la Baie Missisquoi dès 1784 et $\mathrm{y}$ attendirent les faveurs du gouvernement de Québec.

Ils les attendirent longtemps, soit huit ans, parce que le gouverneur Haldimand soupçonnait chez ces nouveaux venus, parmi lesquels s'étaient sans doute glissés des Républicains, des tendances à l'insoumission, en tout cas un esprit démocratique peu compatible avec son régime d'autocratie. Haldimand n'osait mêler à la population canadienne-française ces intrus si différents par leurs us et coutumes, leur langue, et leurs religions variées. Un moment, il songea à les accueillir dans les seigneuries, quitte à déménager les Français le long de la frontière américaine, où ils auraient fait fonction de tampon entre la nouvelle république et le Canada.

A la fin, sous Guy Carleton, successeur de Haldimand, Londres consentit à faire délimiter pour les Loyalistes de Baie Missisquoi des townships dans l'immense espace trapézoïdal de terres restées vagues entre la ligne du $45^{\mathrm{e}}$ et les aboutissants des seigneuries du Saint-Laurent, de la Chaudière et du Richelieu. Les Loyalistes de Baie Missisquoi pénétrèrent en nombre dans ces Eastern Townships dont l'arpentage s'effectua en 1791 et 1792. Notons en passant qu'un township est une division territoriale de 10 milles de largeur sur 10 milles de hauteur. Le terme ne se traduit pas par canton; encore moins par seigneurie. Comme on avait d'abord ouvert des townships à l'ouest (Western), c'est-à-dire à Baie de Quinté depuis 1784, les townships du Bas-Canada, tracés huit ans plus tard, prirent par opposition de situation le nom de Eastern Townships.

Contrairement aux United Empire Loyalists du HautCanada, qui, en plus d'un parlement bien à eux dans les débuts, reçurent huit millions de l'Angleterre pour s'installer en Ontario, nos Loyalistes des Eastern Townships réussirent mal à s'attirer 
les grâces des gouvernants: isolés dans des forêts vierges, à 90 milles du premier marché, celui de Trois-Rivières, sans chemins autres que les rivières, sans écoles, sans temples et sans cimetières, ils accomplirent des prodiges pour survivre à même leurs maigres ressources. Il semble bien que la Clique du Château continuait à redouter ces Américains, à cause sans doute de l'esprit brouillon et entreprenant qu'ils avaient acquis dans leurs petits gouvernements coloniaux; aussi très probablement à cause des croyances baptistes, méthodistes, calvinistes, presbytériennes ou luthériennes que ces hérétiques apportaient avec eux. Ces trouble-fête arrivaient au moment même où le Très Révérend Jacob Mountain inaugurait le premier siège épiscopal anglican à Québec, avec mission de convertir toute la population à la religion du roi. Pour assombrir l'idéal d'unité religieuse et politique du Très Révérend, qu'avait-on besoin de ces dissidents audacieux qui venaient s'ajouter aux tenaces catholiques ?

\section{Exposé de la question}

Quoi qu'il en soit, les attitudes se précisent vers les 1800. Apparemment, quelqu'un dont on ignore le nom jette l'alarme et on se concerte pour établir un plan de grande envergure: les Eastern Townships ne seront ni français ni loyalistes, il faut les britanniser ! De ces Townships, on fera une petite Angleterre, une petite Angleterre farouche, dynamique, et tellement progressive que, non contente de noyer l'élément loyaliste, elle envahira les seigneuries françaises, dont elle repoussera les habitants dans le fleuve. C'est ce plan gigantesque qui m'a inspiré le titre de mon essai, "The Eastern Townships contemplated as a British Stronghold». En 1961, avec quelque 150 ans de recul, on serait tenté de donner à ce titre la traduction libre et gouailleuse qui suit: «Curieuses erreurs de visées de quelques grands politiques $\gg .^{*}$

Que le conquérant ait résolu de bonne heure d'angliciser sa colonie canadienne, il serait bien osé de le contester. Déjà en

* D'avance je proteste de tout mon respect pour mes compatriotes actuels d'expression anglaise. J'ajoute que leurs ancêtres, les politiques britannisants, caressaient un rêve qui ne manquait pas de vraisemblance, et que d'autres conquérants tels que les Romains régnant sur la petite Grèce de 146 A.C., ont entretenu avec la même ferveur, comme avec le même insuccès. 
1763, en dépit des clauses des capitulations de Québec et de Montréal et de celles du traité de Paris qui nous garantissaient la préservation des traditions et la pratique du culte, des «Instructions secrètes » parvenaient de Londres à Murray, après la Proclamation royale, indiquant les procédés à suivre pour anglicaniser les Canadiens. Tout particulièrement les articles 32 , 33 et 38 de ces Instructions ordonnaient comme suit:

32. - You are not to admit of any Ecclesiastical Jurisdiction of the See of Rome, or any other foreing Ecclesiastical Jurisdiction whatsoever in the Province under your Government.

33. - An to the End that the Church of England may be established both in Principles and Practice, and that the said Inhabitants may by Degrees be induced to embrace the Protestant Religion, and their children be brought up in the Principles of it; We do hereby declare it to be Our Intention, when the said Province shall have been accurately surveyed, and divided into Townships, Districts, Precincts or Parishes, in such manner as shall be hereinafter directed, all possible Encouragement shall be given to the erecting Protestant Schools in the said Districts, Townships and Precincts, by settling, appointing and alloting proper Quanties of Land for that Purpose, and also for a Glebe and Maintenance for a Protestant Minister and Protestant School Masters; and you are to consider and report to Us, by Our Commissioners for Trade and Plantations, by what other Means the Protestant Religion may be promoted, established and encouraged in Our Province under your Government.

38. - And We do further direct, that no Schoolmaster who shall arrive in Our said Province from this Kingdom, be henceforward permitted to keep School, without the Licence of the said Lord Bishop of London; and that no other Person now there, or that shall come from other Parts, shall be admitted to keep School in your Government, without your Licence first obtained. ${ }^{1}$

${ }^{1}$ W. P. M. Kennedy, Documents of the CANADIAN CONSTITUTION, (Toronto, University Press, 1918), 27: Instructions to Governor Murray, 7 December 1763. 
Ces instructions, même si les circonstances ont empêché qu'elles ne soient appliquées à la lettre, et même si on en a modifié la rédaction de temps à autre, ont été maintenues sous chacun des successeurs de Murray.

$\mathrm{Vu}$ ce parti pris avoué, on n'a pas à se surprendre de ce que les Loyalistes, même s'ils étaient anglophones, n'aient guère eu plus de chance de se faire agréer avec leurs conceptions puritaines (leurs ancêtres, à cause de leur anti-anglicanisme, avaient dû fuir l'Angleterre), et leurs tendances démocratiques (ces Loyalistes s'étaient instruits sur leurs droits de citoyens au cours de la Guerre d'Indépendance) : c'étaient des sujets encombrants, à qui il fallait rendre l'existence difficile, parce qu'ils menaçaient de brouiller un beau rêve d'unité religieuse et politique. C'est, nous l'insinuons de nouveau, ce qui détermina les autorités à prendre des mesures pour que les Eastern Townships, qu'on avait copieusement baptisés de noms empruntés à la toponymie de la Grande-Bretagne, ne devinssent ni français ni loyalistes, mais férocement britanniques.

Quelques faits révélateurs et quelques témoignages éloquents nous permettent d'illustrer cette radicale prise de position.

\section{Les faits}

$1^{\circ}$ - Dès 1800, le Comité des Terres du gouvernement de Québec, qui avait octroyé jusque-là des «lots » de la Couronne aux Loyalistes dans les Eastern Townships, change carrément d'attitude après la loi du 4 août: il cesse la concession de terres de la Couronne à des Loyalistes. Il n'est pas non plus question d'inviter les Canadiens français à coloniser ce paradis: la première concession de terres de la Couronne à un riverain français $\mathrm{du}$ Saint-Laurent retardera longtemps. ${ }^{2}$ Non, c'est à des citoyens britanniques, ou de sentiment britannique, que le Comité des Terres réserve désormais ses dons gratuits. Plus encore, on dépossédera des Loyalistes déjà installés dans les Townships pour avantager de leurs propriétés défrichées des amis ou des membres du conseil législatif: Isaac Ogden, juge de l'Amirauté, Henry Coldwell, receveur de la province, Shore Milnes, lieute-

2 Voir J.-C. Langelier, Liste des Terrains concédés de 1791 à 1890. 
nant-gouverneur, comptent parmi ces favoris qui, délogeant des Loyalistes sous quelque prétexte légal, s'approprieront leurs terres prometteuses.

$2^{\circ}$ - Les Eastern Townships deviennent, aussi, un objet de pécule et d'agiotage, au profit de sympathisants britanniques seulement. Les exemples qu'on relève montrent la frénésie avec laquelle les amis du gouverneur se jetèrent sur des étendues immenses qu'ils obtenaient sans bourse délier et sur lesquelles ils se proposaient de spéculer : ${ }^{3}$

\begin{tabular}{lllrl}
\multicolumn{1}{c}{ Concessionnaire } & \multicolumn{1}{c}{ Fonction } & \multicolumn{1}{c}{ Township } & Acres & Date \\
John Black & Député de Québec & Dorset & 53,000 & 1797 \\
John Jones & Marchand de Québec & Huntertown & 24,622 & 1800 \\
William Grant & Conseiller législatif & Grantham & 27,000 & 1800 \\
Hugh Finlay & Conseiller exécutif & Stanbridge & 41,700 & 1800 \\
Rvd Philip Toosey & Ministre anglican & Stoneham & 24,000 & 1800 \\
Simon McTavish & Commerçant & Chester & 15,000 & 1802 \\
Benjamin Jobert & Commerçant & Halifax & 15,000 & 1802 \\
William McGillivray & Commerçant & Inverness & 15,000 & 1802 \\
Isaac Todd & Commerçant & Leeds & 15,000 & 1802 \\
John Gregory & Commerçant & Arthabaska & 15,000 & 1802 \\
Joseph Frobisher & Commerçant & Irelant & 15,000 & 1802 \\
David Alex. Grant & Neveu de William & Upton & 25,200 & 1802 \\
William Lindsay & Assemblée législative & Wickham & 23,753 & 1802 \\
Thomas Scott & Contrôleur des douanes & Durham & 21,991 & 1802 \\
George Langmore & Médecin garnison Québec & Kingsey & 11,418 & 1802 \\
Henry Coldwell & Receveur de la province & Westbury & 12,262 & 1804 \\
Henry Coldwell & Receveur de la province & Melbourne & 26,153 & 1805 \\
Joseph Frobisher & Commerçant & Divers & 57,000 & 1810 \\
Shore Milnes & Gouverneur général & Stanstead & 21,406 & 1810 \\
Shore Milnes & Gouverneur général & Divers & 50,000 & 1812 \\
Thomas Dunn & Membre Comité des Terres Stukely & 11,600 & 1816 \\
William Osgoode & Juge en chef & Sutton & 12,000 & 1817 \\
W. B. Felton & Officier de la marine & Ascot & 26,500 & 1822 \\
B. A. Land Co. & Compagnie de Londres & Divers & $1,094,272$ & 1834 \\
& &
\end{tabular}

$3^{\circ}$ - On commence tôt à orienter vers les Eastern Townships des immigrants, mais des immigrants d'esprit ou d'origine

3 Après l'enquête de Buller, en 1838, Lord Durham lui-même se scandalisera de ce que 105 propriétaires détiennent à eux seuls environ $1,500,000$ acres de terre dans les Townships (J.-C. Langelier, Liste des Terrains concédés entre 1791 et 1890, Préface).

4 Bouchette, Topographical Dictionary of Lower Canada, voir liste à la fin du volume; voir aussi J.-C. Langelier, Liste des Terrains concédés. 
britannique, pas des Loyalistes ni des Français; notamment après 1812, une fois terminées les guerres napoléoniennes, on dirigea vers les Townships des officiers ou soldats licenciés des armées de la Grande-Bretagne, et ce mode de peuplement prit assez d'ampleur pour que les sujets de provenance anglaise dépassassent bientôt en nombre les Loyalistes restés agrippés à leurs propriétés. C'est à ce moment qu'apparaissent les noms de Felton, Beckett, Whitcher, Terrill, Fletcher, Bowen et autres.

$4^{\circ}$ - En 1818, l'ancien Ktiné, qui s'appelait Hyatt's Mill depuis 25 ans, ne mérite plus de porter le nom du fondateur loyaliste Gilbert Hyatt: les notables du hameau, en tête William Bowman Felton, intercèdent auprès du gouverneur du temps pour qu'on rebaptise l'endroit d'un nom qui sonne vraiment londonien, et Lord Sherbrooke condescend à donner son nom à la future métropole des Eastern Townships. ${ }^{5}$ Cette simple conversion d'une appellation porte tout un sens. La mode se propage, et plusieurs localités naissantes gagnent aussi de s'orner d'une désignation qui les apparente à la cour royale: Drummondville (en l'honneur du gouverneur Sir Gordon Drummond), Lennoxville et Richmond (en l'honneur de Charles Lennox, duc de Richmond, gouverneur), Charleston (en l'honneur du Prince Régent Charles), Aylmer (en l'honneur du gouverneur Lord Matthew Aylmer), Victoria (en l'honneur de la grande reine), Georgeville (en l'honneur du roi George).

$5^{\circ}$ - Le projet d'une «Union des deux Canadas», Haut et Bas, a mijoté de bonne heure en quelques esprits: avant plusieurs autres personnages en place, John Black, un théoricien finaud et un profiteur de haute classe, le propose dès 1806 comme un procédé merveilleux pour remédier aux «maux de son temps ${ }^{6}$ L'idée fit son chemin; elle paraît avoir enthou-

5 The Quebec Gazette, 8 février 1818.

6 "The Remedies which I would suggest for the Evils classed under the different Heads is to unite the Provinces of Upper and Lower Canada if it conveniently can be done, if not erect eight new Counties in the three Million acres of Land recently granted who would return two Members each. ... The uniting the Provinces or erecting eight new Counties would bring the English considerably nearer to the French in point of Number. Etc." Kennedy, Documents of the Canadian Constitution, 247, Observations on the Government of Canada by John Black, 9 Oct. 1806. 
siasmé les résidants des Eastern Townships: coincée entre deux blocs anglophones, celui de l'Ontario (Western Townships) et, à condition de la renforcer, celui des Eastern Townships, la population des Canadiens français deviendrait une proie facile à réduire à néant. Aussi, en 1822, après les recommandations à Londres de Ellice, une requête circule dans les Eastern Townships, et elle se couvre de 10,000 signatures. Pour mesurer l'ardeur des pétitionnaires, on notera que cette requête trouve 10,000 signataires seulement dans un milieu qui ne comptait pas alors 40,000 anglophones: pour atteindre ce chiffre, il a vraisemblablement fallu faire signer jusqu'à des gosses de dix ans. Nous reproduisons plus bas des extraits de ce document où on demande en somme de faire des townships, par l'immigration et par l'union des deux Canadas, une «honoured resemblance of the Parent State ».

$6^{\circ}$ - En même temps, des résolutions partent pour Londres, formulées par des membres anglais du Conseil législatif du BasCanada; parmi les signataires, William Bowman Felton, officier de grande influence représentant Sherbrooke. Ce document aussi propose, en plus de l'union des deux Canadas, l'intensification de l'immigration britannique, afin de donner au Bas-Canada un caractère vraiment «British 》.

$7^{\circ}$ - En compulsant les correspondances des premiers missionnaires, tels Jean Holmes, Hugh Paisley, Hugh Robson, J. B. MacMahon, on retrouve à l'occasion des allusions à l'espèce d'opposition qui subsiste entre les Britanniques et les autres résidents de la région, particulièrement les «Américains 》 (lire les Loyalistes). L'abbé Jean Holmes, dans une lettre adressée à l'évêque de Québec le 21 septembre 1824, mentionne que Monsieur Felton, un Anglican dont l'épouse et les filles sont catholiques, semble disposé à contribuer à l'érection d'une chapelle dans le voisinage de sa demeure, mais, note-t-il aussitôt, «comme les gens de là sont des Américains, ils n'aimeront pas trop déprendre de lui ». Le 19 novembre de la même année (autre lettre à l'évêque de Québec), le missionnaire hésite entre une double offre de terrain pour l'église, offre faite par Monsieur Felton et Monsieur Goodhue, ce dernier un «Américain», et 
l'abbé Holmes est embarrassé, parce que, les deux n'appartenant pas au même clan, s'il accepte l'avance de l'un, il indisposera l'autre. L'abbé Michael Power (lettre à l'évêque de Québec le 10 août 1828) est plus explicite:

Le gouvernement a pour but de faire de ces townships le centre du protestantisme, et par une contradiction assez singulière, on voudrait $y$ voir que des Canadiens afin de forcer les Irlandais et les Ecossais à s'en aller dans le Haut Canada et pour fermer toute entrée aux Américains. Les terres se concèdent rapidement et les Canadiens refusent d'en prendre parce qu'ils se trouvent trop éloignés des Eglises et des prêtres. S'ils voyaient que cette Eglise s'achevait, ils prendraient courage, s'y établiraient et empêcheraient par là que toute cette partie du pays fût occupée par des Protestants. On ne compte pas moins de cinq ministres anglicans, sans parler d'un qui est interdit...

Encore au temps de l'abbé J. B. MacMahon, premier curé (1834-1839), on fait des distinctions (sa correspondance en témoigne) entre le descendant du Loyaliste, cet Américain suspect et toléré, et l'Anglais de souche, fier et bien racé... ${ }^{7}$ C'est peut-être le juge John Fletcher, un importé d'Angleterre (il présida le tribunal du district Saint-François de 1822 à 1842) qui personnifie le plus crûment l'arrogance des siens devant la portion plus modeste, parce que non britannique, de la population confiée à sa juridiction: il vante à hauts cris la loi anglaise et se moque du code de la province, qu'il est pourtant chargé d'appliquer; il se montre méprisant et injuste envers les prévenus ou témoins loyalistes qui passent devant lui; il les traite sans vergogne de «wild beasts of the forest»; il les condamne et les emprisonne sans merci. Heureusement, ses congénères n'extravaguent pas tous avec autant d'éclat. ${ }^{8}$

$8^{\circ}$ - Depuis 1791, un seul comté, celui de Buckinghamshire, embrasse presque tous les Townships. Les britannisants insistent pour qu'on subdivise ce comté en plusieurs districts élec1832.

7 Archives des archevêchés de Québec et de Sherbrooke.

8 Rapport du Comité permanent des Griefs, Gouvernement Québec, 
toraux, afnn d'augmenter leur représentation à la Chambre, en majorité française. Commencée vers 1810, la campagne réussit en 1829, alors que le gouvernement taille du coup huit comtés dans Buckinghamshire. A deux représentants par nouveau comté, dans une Chambre qui contenait 50 députés dont 16 déjà d'appartenance anglaise, le parti anglais accroissait considérablement son poids dans les discussions et décisions parlementaires.

$9^{\circ}$ - En 1833, Londres approuve la fondation d'une compagnie de colonisation, la British American Land Company, dont Alexander Galt deviendra le commissaire en chef, et dont la première préoccupation comme le premier but avoué sera de faire venir des Iles Britanniques des flots d'immigrants.

$10^{\circ}$ - Durant tout ce temps, les Canadiens français, qui étouffent dans leurs seigneuries laurentiennes à cause de leur multiplication (de 60,000 en 1760 , ils sont passés à 600,000 en 1836), lorgnent inutilement vers les terres des Townships, détenues par des magnats. Sans doute la tenure libre (free and common soccage) instituée dans la région neuve les fait hésiter à s'y engouffrer, parce qu'ils la connaissent peu ; mais la raison de fond qui les retient, c'est toujours l'espèce de conspiration qui rend difficile à d'autres qu'à des anglophones l'acquisition d'une propriété dans cette chasse gardée. Quelques riverains du fleuve, après 1827, s'infiltrent courageusement dans Stukely, Kingsey, Drummond, Stanfold, mais c'est pour y subir des sévices et des épreuves. A Sherbrooke même, vers 1834, on ne compte qu'une quarantaine de noms français; et ce sont non des propriétaires, mais des manœuvres et des serviteurs; l'industrie, le commerce et le prestige restent aux mains de Britishers, et surtout de la $B$. A. Land Company. ${ }^{9}$ C'est cette année-là que, persuadés qu'on les ostracise, les députés de la Chambre proposent les «92 Résolutions», dont l'une proteste contre la mainmise des Britanniques sur les Townships: la Résolution $84,7^{\circ}$ s'élève contre le système suivi pour la concession des terres de la Couronne depuis 40 ans, et elle rappelle que des personnages

${ }^{9}$ Les premières firmes canadiennes-françaises, comme celle de Codère et Fils, ne se fondèrent dans la région que vers 1865 . 
de haut rang, d'appartenance britannique, de connivence avec leurs amis du gouvernement, ont frauduleusement spéculé sur les terres vagues et les ont monopolisées au détriment de la majorité (française) du peuple. ${ }^{10}$ On pourrait mettre en doute les allégations des auteurs des «92 Résolutions», à cause de l'aigreur qui perce dans tout le document. On devra alors, pour se convaincre du bien-fondé de leurs récriminations sur l'attribution des terres, s'en remettre au témoignage de Lord Durham qui, sur le rapport de son enquêteur Charles Buller en 1838, confirme l'existence d'un exécrable trust sur le domaine de la Couronne. Au témoignage aussi d'Antoine Racine et de ses douze collègues, exposé dans «Le Canadien émigrant », mémoire paru en 1851, les précisions et les exemples d'abus abondent, en la région. D'après ces deux sources sûres, il semble bien que tout le siècle dernier a résonné du slogan « No Frenchmen need apply ».

$11^{\circ}$ - Lorsqu'en 1837 le soulèvement éclate, on s'émeut, bien inutilement d'ailleurs, à Hatley, à Compton et à Sherbrooke: on appréhende une invasion, moins par Granby que par Stanstead, où Papineau a soudoyé puis lié à son parti un certain nombre d'Américains des deux côtés de la frontière. A Sherbrooke notamment, on organise une milice active, pro-britannique évidemment, qui se tient aux aguets, carabine au poing; cependant que le curé John Baptiste McMahon, dans un faux élan d'opportunisme et de zèle, multiplie les sermons d'une ou de deux heures, à Sherbrooke, à Tingwick et à Kingsey, sur le devoir d'obéissance à l'autorité royale. ${ }^{11}$ C'est le rebelle français qu'on redoute, mais des descendants de Loyalistes semblent aussi disposés à participer à la révolte: ne sont-ce pas justement des fils de Loyalistes qui, au même temps, mènent la révolution dans le Haut-Canada ?... A la vérité, ce sera la dernière fois que nos Britanniques éprouveront des soupçons sur la fidélité des Loyalistes des Eastern Townships: l'antipathie des premiers temps s'est estompée, les Loyalistes de la troisième génération,

10 Kennedy, Documents of the Canadian Constitution, The Ninety-two Resolutions, 385.

11 Archives, archevêchés de Québec et de Sherbrooke. 
en partie assimilés par leurs congénères anglais, feront désormais cause commune avec eux sous l'Union, et l'épouvantail n'apparaîtra plus que du côté des Français ... Aussi, pour revigorer la thèse des jours de 1800, deux fois coup sur coup après 1837, Alexander Galt passera en Angleterre pour mousser de nouveau à grands renforts de propagande et d'embauchage, l'immigration massive, dans les Townships de l'est, des plus valeureux colons de la Grande-Bretagne. ${ }^{12}$

$12^{\circ}$ - L'université Bishop, à Lennoxville, surgit en 1848. Avec sa charte royale et son inspiration anglicane, elle ne donne l'impression de vouloir ni raviver l'élément loyaliste, qui d'ailleurs a perdu alors son caractère distinct, son nombre et son prestige, ni favoriser l'élément français et catholique qu'on espère toujours garder à distance. Je m'étonne de ce qu'on ait pu avoir une université protestante et anglaise dans les Townships avec une population de 50,000 anglophones, alors que les Canadiens français ont dû attendre, dans la même région, de se nombrer à environ 800,000 pour mériter une université française et catholique à Sherbrooke, en 1954 seulement. L'université Bishop a été durant tout ce siècle et reste aujourd'hui encore un foyer actif et conscient de culture anglaise et de doctrine anglicane.

$13^{\circ}$ - Alexander Galt, un Sherbrookois, et un des pères de la Confédération, semble avoir, un des premiers, entrevu un revirement possible dans les événements. Forcé lui-même, par le succès insatisfaisant de l'immigration britannique, d'accueillir à contrecœur des colons francophones sur les terres de la British American Land, compagnie qu'il dirigeait, il paraît s'être affolé à la pensée que l'introduction de sujets français dans les Townships pourrait bien un jour changer la carte électorale et diminuer ainsi le nombre de députés anglophones à la Chambre. On raconte donc qu'une fois rendu à Londres avec le projet de l'Acte de l'Amérique du Nord britannique, c'est lui qui fit ajouter à l'article 80 , et de son propre chef, sans consulter l'assemblée des Pères, une clause qui traduit un effort suprême pour garder

12 Oscar Douglas Skelton, Life and Times of Sir A. T. Galt. 
aux comtés des Townships des représentants de sa race. La clause stipule que si jamais on médite de modifier la délimitation des districts électoraux dans les Townships, ${ }^{13}$ il faudra requérir un vote non seulement de la majorité à la Chambre, comme à l'ordinaire, mais aussi un vote spécial des $2 / 3$ des députés de ces districts électoraux, plus une adresse au lieutenant-gouverneur certifiant qu'on a bien pris le soin de prendre un vote spécial. Cette clause, pleine d'astuce, n'aurait pas sa raison d'être si elle ne cachait un ultime désir de conserver dans les Eastern Townships une influence nettement anglaise.

On fausserait la vérité en affirmant qu'avec Galt disparut à jamais l'ambition de britanniser les Cantons de l'Est et par là le reste de la province: le rêve s'effiloche depuis 100 ans, mais il n'est pas complètement mort, on pourrait le démontrer par des faits actuels. Il ne me paraît pas utile toutefois, pour la valeur de mon exposé, de relever les initiatives tentées périodiquement depuis 1867 pour se raccrocher aux desseins fantastiques de Hermann Witsius Ryland. Qu'il suffise d'invoquer, pour la période 1800-1867, quelques témoignages qui confirment l'intention dont nous semblent animés les faits déjà rapportés.

\section{Témoignages}

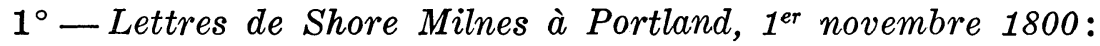

I am well aware the chief Object to be depended upon to encrease ${ }^{14}$ the Influence of the Crown, will be by means of the Waste Lands $;^{15}$ and in that point of view the delay that has taken place in the Land Business in greatly to be regretted and it becomes an Object of peculiar importance to Government that no further delay may occur to prevent the clearing and settling of the immense Tracts that are now in the hands of the Crown indisposed of, as their being granted in free and common Soccage

13 Les comtés impliqués sont Pontiac, Ottawa, Argenteuil, Huntington, Missisquoi, Brome, Shefford, Stanstead, Compton, Wolfe et Richmond, Mégantic et la ville de Sherbrooke.

14 Dans cette citation comme dans les autres, nous reproduisons le texte aussi fidèlement que possible, avec, lorsqu'il en contiendra, les bizarreries de graphie ou de syntaxe.

15 Chaque fois qu'il sera question de Waste Lands, ou de terres vagues, bien comprendre qu'il s'agit des Eastern Townships. 
will in time (if judiciously granted) form in this Province a Body of People of the Protestant Religion that will naturally feel themselves more immediately connected with the english Government; but as this cannot be expected to have any immediate Effect, I am inclined to think that in the mean time much may be done first through the catholic Priests, and secondly by means of the militia. ${ }^{16}$

Dans la suite de sa longue lettre, Milnes propose done de tâcher de circonvenir l'évêque catholique et son clergé en leur offrant de l'argent! Par le même moyen, on gagnerait aussi la 《loyauté » des capitaines de milice canadiens-français, et leur zèle total pour des initiatives nettement pro-royalistes.

Le « if judiciously granted » qu'on a lu plus haut est suave, quand on sait qu'à partir de Milnes, seuls des favoris britanniques ont reçu des terres dans nos Townships. Milnes précise que sur les 150 townships disponibles et déjà sollicités, 35 ont déjà leurs propriétaires: l'attribution . . . judicieuse des 115 autres accroîtra la richesse, le pouvoir et le prestige de la Mother Country.

$2^{\circ}$ - Portland à Milnes, 6 janvier 1801.

Portland entre dans toutes les vues de Milnes: acheter la complaisance des capitaines de milice, réduire les prétentions du clergé catholique et lier l'évêque par de forts émoluments. Il ajoute:

These leading points relative to the Roman $\mathrm{Ca}$ tholick Clergy and the militia being carried, every future step which is made in the settlement of the Province must, by making grants of the Waste Lands of the Crown to Protestants upon the conditions, and subject to the Regulations now finally established and acted upon in the Land Granting Department, necessarily tend to lessen the degree of popular influence which is at present possessed by that description of His Majesty's Canadian subjects which constitutes so great a proportion of the inhabitants of the Province at large. ${ }^{17}$

16 Kennedy, Documents of the Canadian Constitution (1918), 241.

17 Ibid., 244. 
Dieu ! qu'il lui en faut des mots, à ce Portland, pour affirmer tout simplement qu'on devra affaiblir et mater les Canadiens français: «Every future step... must necessarily tend to lessen etc. »

$3^{\circ}$-John Black au duc de Kent, 9 octobre 1806.

The Remedies which I suggest for the Evils classed under the different Heads is to unite the Provinces of Upper and Lower Canada if it conveniently can be done, if not erect eight new Counties on the three Million acres of Land recently granted who would return two Members each to make the Parliement sit for seven years in lieu of four years, and render a qualification necessary for every Member to the extent of at least one hundred and fifty pounds $\mathrm{p}$ Annum in landed property, or a permanent Salary to that amount - I should wish here to observe to your Royal Highness, the impossibility the Country can prosper under the present constitution which draws forth a majority of the most inflamed of the worser order, whilst the qualification proposed would draw forth the Sense and Education of the Country. ${ }^{18}$

On aura saisi l'arrière-pensée du roué John Black. Le peuple élisait à la Chambre de nombreux députés canadiensfrançais pour leur personnalité bien plus que pour leur pauvre avoir: ils n'avaient pas le sou. Un moyen habile de se défaire d'eux serait d'exiger, aux élections, un cens d'éligibilité excessif, en propriété ou en argent. De nombreux pro-britanniques, en possession des terres, du commerce et de l'industrie, pourraient alors bloquer la voie à leurs adversaires. L'idée de base: il faut arriver à donner aux «British born subjects» la prépondérance à la Chambre. La création de huit nouveaux comtés dans les Townships favoriserait ce dessein.

$4^{\circ}$ - Herman Witsius Ryland, Observations sur l'état politique du Bas-Canada, mai 1808.

L'auteur démontre que le gouverneur, avec l'appui du Conseil exécutif, et done sans se soucier de l'approbation de la 18 Ibid., 246. 
Chambre, devrait proclamer en décrets les mesures suivantes que lui, Ryland, préconise: augmentation des représentants anglais à la Chambre et dans l'Exécutif ; même chose pour la milice et la judicature; les juges puînés, nommés sur la recommandation du juge en chef, siégeraient (au moins un tiers d'entre eux) à la Chambre; le tout pour diminuer l'influence française. Il veut aussi multiplier les comtés dans les Townships, en assignant un député à chaque 1,000 habitants; ces députés seraient anglais, et ils présenteraient des titres de propriété foncière prohibitifs pour les Canadiens français. En conclusion:

Such an Act would not only encourage the settlement of the waste lands, and attach the inhabitants of the townships to His Majesty's Government, by enabling them to participate in the legislation of the Province, but it would provide and effectual check upon the French or Roman Catholic party, which has at this time an alarming preponderance. ${ }^{19}$

\section{$5^{\circ}$ - Craig à Liverpool, Québec $1^{\text {er }}$ mai 1810.}

Le bilieux gouverneur n'est pas tendre pour les Canadiens français: il les déteste souverainement. A son avis, on devra éliminer de la Chambre ceux qui les représentent, et remplacer ces vauriens par des hommes doués d'honneur, de moyens, d'éducation, des britanniques, quoi ! On s'occupera aussi de remplir les Townships d'anglophones, qui enverront des anglophones au parlement. A défaut d'Anglais authentiques, on se résignera à introduire dans les Townships des Américains, même si on entretient quelque soupçon sur leur aptitude à la fidélité: au moins, ce ne sont pas des catholiques; et avec le temps, ils se britanniseront:

The great object of their jealousy at this moment is, the progress of the Townships - that is, in fact the introduction of Settlers of any denomination but Canadians, as having a tendancy, which of all others, they are most anxious to assert, to impede the complete Establishment of a Canadian Nation; These Townships are generally settled by Americans, a proportion of whom are Loyalists who were under

19 Ibid., 248. 
the necessity of quitting their Country on the peace of 1784, but by far the greater Number are of Americans who have come in and settled upon those lands since that event. How far it may be good policy to admit of settlers of this description is another question, the Canadians however are loud in their Clamours against it; The circumstance of being Americans, and the principles generally attributed to these, afford them the pretext, while the truth is, it would be equally repugnant to the idea they entertain of their own Interests, and they would just have the same feelings upon it, were the tract in question in a progress of settlement from Britain and Ireland ... This jealousy has increased much since they are become more systematic in their operations, and will now totally prevent any measure that may be proposed for the benefit of that part of the Country...

Nest to this great measure, that which is most generally looked up to, is the Reunion of the two Provinces...

It has been suggested that by a new Division of the Province new Counties might be formed in that part now distinguished by the general name of the Townships, from whence Members might be furnished with the same view of Balancing the Canadian Party; this seems to me more practicable, at least than the proposed re-union of the Provinces, besides being in itself a measure that is in some sort required in Justice to the Inhabitants, who begin to complain of not being represented: the Canadian part of the Electors so infinitely outnumber them tho' confined to a much less extent of Country that they can never succeed...

The qualification that I think best adapted to the circumstance of the Country, would be one hundred pounds Currency, clear annual revenue arising from Land actually the property of the person presenting himself, for twelve Calendar Months previous to the day of election, or two thousands pounds Currency in personal property clear of all debts or demands... ${ }^{20}$

20 Ibid., 256 et seq. 
$6^{\circ}$ - Observations du juge Sewell sur l'union des deux provinces (1810?)

Pour qui aime le style cauteleux et les préjugés profonds, tout le mémoire du juge Sewell est à lire. Ne pouvant ici en citer que des extraits, nous renvoyons à la source le lecteur qui voudrait se délecter de ce morbide réquisitoire contre l'élément français. Lui aussi en veut à la juridiction de l'évêque catholique; lui aussi favoriserait l'imposition d'écoles protestantes et anglaises dans les seigneuries; et lui aussi souhaite l'union des provinces, l'accroissement du nombre des députés anglophones et le peuplement massif des townships. A noter que le juge Sewell n'a pas toujours chéri les relations avec les Etats-Unis: c'est lui qui, une dizaine d'années auparavant, a condamné l'Américain David McClean, et, pour effrayer tous ses congénères tentés de venir espionner au Canada, l'a fait pendre sur la place publique à Québec, puis l'a fait dépecer vivant pour semer ses membres et ses entrailles aux quatre coins de la ville. Mais Sewell est un opportuniste: aujourd'hui, « to sink the Canadian population » et à défaut de «British born subjects», il inviterait volontiers des multitudes d'《American born subjects » à passer dans les Townships !

... Yet the Province must be converted into an English Colony, or, it will ultimately be lost to England.

I am led from these considerations in the first instance to conceive it indispensably necessary to overwhelm and sink the Canadian population of (Sic) English Protestants, and this I believe to be practicable; I do not mean that subjects can or ought to be procured from England to the extent required for this purpose, but they may, and I think ought to be procured from the neighbouring States, For although it may be feared by some that they would not be good Subjects, I have myself no such fears; I believe that once settled in the Province they would have no wish to return to their former system of Government, an expectation justified by the conduct of those who are already settled in the country. It is besides only in the case of a War with the Northern States of America that 
the disaffection of such settlers is to be dreaded, and this is an event to be contemplated as a remote contingency ... ${ }^{21}$ Such settlers it is certain would be the descendants of Englishmen, profess the same religion, ${ }^{22}$ and speak the same language, and would therefore be more easily assimilated, and become better subjects than those which we now possess, and if to people the Country with such Characters is to incur a risk, the risk incurred will be less than that which we must incur by suffering the Province to remain in its present state.

The Waste Lands of the Crown afford sufficient means for the accommodation of a much greater number of Settlers than is required, But their dispersion through the settled parts of the Country is desirable upon many accounts, and to effect this would require the aid of Parliament. All the Grants of the French Government were made under the feudal System, and all the lands so granted, are now so held by the Lords of the several Seigneuries in Canada, and their respective Tenants. To such Tenures all Englishmen and Americans have an utter aversion, and the consequence is that all the Seigneuries in the Province are entirely settled by Canadians. Most of the Seigneurs however, would be glad, to take a fixed price for the fee simple of their farms, and in consideration of that Price to exonerate them from the payment of all rents, mistaken fines; and other Feudal Burthens for ever...

In the present state of the Legislature of Canada, three fourths of the House of Assembly are Canadians, and of that proportion of the whole nearly of the lowest Class, The fruits of Universal Suffrage, Four fifths of the whole also are Roman Catholics ... The Introduction of English Settlers of itself will increase the number of English Members but the augmentation of their number would be greatly promoted by an Act requiring a qualification as well for Members as for Electors. The Character of Canadians is Idleness and inactivity, of the English Settlers Industry and perseverance... Generally

$21 \mathrm{La}$ guerre entre le Canada et les Etats-Unis devait éclater moins de deux ans plus tard!

22 Ceci nous paraît bien loin de la vraie probabilité. 
speaking therefore, the English Settlers will possess property of greater value than the Canadians, and if qualifications comparatively high are required, The nomination of Members to the Lower House will ultimately rest with the English Settlers. The number also of Persons qualified to be Members will increase among them, while among the Canadians it will be diminished...

... in my mind, none (measures) would be more efficacious than an incorporate union of the two Provinces of Upper and Lower Canada under one Governor General, and one Legislature ...23

$7^{\circ}$ - Rapport de H. W. Ryland au secrétaire d'Etat Peel, 11 février 1811.

Ryland résume les propositions qu'au nom de Craig il a portées récemment devant le Parlement de Londres. Le $3^{\circ}$ de son rapport se lit comme suit:

The adoption of a system for the more speedy settlement of the waste lands of the Crown, by which means the English population, the agriculture, commerce, and revenues of the Province might be rapidly increased..$^{24}$

$8^{\circ}$ - Mémoire au soutien de la requête des Habitants du BasCanada (1814?)

... Ceux du parti anglais sont opposés à leurs (Canadiens) intérêts, en ce qu'ayant beaucoup plus d'affinité avec les Américains par leurs mœurs, leur religion, leur langage, ils encouragent la population américaine, comme un moyen de se débarrasser des Canadiens qu'ils regardent toujours comme une population étrangère, comme une population française et Catholique, avec les mêmes préjugés que la classe du peuple, dans la mère-patrie, a contre les Français et les Catholiques, ils ne peuvent s'empêcher de se regarder comme dans un pays étranger, dans une province où la population canadienne (française) domine; une colonie peuplée d'Américains leur paraît plus une colonie anglaise, et ils ne s'y regarderaient pas autant comme dans un

23 Ibid., 267 et seq.

24 Ibid., 281. 
pays étranger. Ces effets sont encore augmentés par la circonstance que la plus grande partie, peutêtre, des officiers du Gouvernement est devenue personnellement intéressée à l'introduction de la population américaine dans ce pays, par les concessions des terres de la Couronne, qui leur ont été accordées, dans le voisinage des Etats-Unis; ainsi, le parti anglais est opposé au parti canadien, justement sur le point qui touche à sa vie et à son existence comme peuple...25

L'avis des Habitants diffère ici de celui des Anglais en général sur l'accueil réservé à d'éventuels immigrants américains : comme on l'a vu par les témoignages de Craig et de Sewell, c'est en désespoir de cause qu'on implanterait des Américains dans les Townships ... Par contre, la dernière phrase de l'extrait que nous venons de citer résume bien, croyons-nous, l'enjeu de la partie: l'élément britannique veut à tout prix s'ancrer, race et mentalité, dans les Eastern Townships et dans tout le BasCanada.

$9^{\circ}$ - Requête des citoyens des Eastern Townships en faveur de l'union (1822).

... The townships or English Lower Canada are people wholly by Inhabitants of British birth and descent and American Loyalists amounting at present to about 40,000 souls, who have no other language than that of their British ancestors, who inhabit Lands granted under the British tenure of free and common soccage, who have a Protestant clergy for whose maintenance a portion of whose lands is set aside, and who, notwithstanding, are subject to French Laws (the custom of Paris) of which they know nothing, compiled in a language with which they are unacquainted... ... Your Petitioners will not enlarge upon the general statement they have given of their condition, by entering into the details of the numerous hardships and difficulties with which they have had no contend tho' sensible that the recital would call forth commiseration. They will content themselves with

25 Ibid., 286. 
stating, that as settlements under these English Tenures have been commenced, as immense tracts still remain to be settled, and as the population of Lower Canada is trifling compared to the amount which it is capable of attaining, there can be no sound reason for rearing up any portion of the Province, so as at its maturity to constitute a nation of foreigners or for continuing a system calculated to deter Britons and their descendants from settling upon the waste Lands of the Crown ... ... Already within a recent period nearly a hundred thousand Emigrants of British birth have made Lower Canada a place of transit, who, if the foreign aspect of the Legislature had not urged them to take an abode elsewhere, might have augmented strength and means of the English population in the province. But notwithstanding the past checks to colonial increase, unless similar causes are allowed to operate hereafter, future Emigrants and their descendants joined to the English already established here, may ultimately form a great majority of the inhabitants, and render the country in fact as it is in name, a British Colony... .... a people who should perpetuate in after ages the honoured resemblance of the Parent State... and while the freedom and protection of Britain are bestowed upon Canadians it can neither be unfair nor ungenerous to require in return the existence of such an amended constitution as shall encourage a portion of our Brethren from Britain, to establish themselves and their posterity upon the Crown Lands in Lower Canada .... ${ }^{26}$

La requête se termine par deux demandes: l'Union des deux Canadas et l'augmentation de la représentation des Townships au Parlement.

$10^{\circ}$ - Résolutions de la minorité du Conseil législatif du BasCanada (1822).

Six signatures anglaises, parmi lesquelles celle de Hermann Witsius Ryland, dont nous connaissons déjà le sentiment, et celle de William Bowman Felton, de Sherbrooke, alors membre du Conseil législatif. Ces résolutions s'appuient des motifs suivants:

26 Ibid., 326. 
$1^{\circ}$ - L'Union mettrait fin aux difficultés fiscales et budgétaires, ainsi qu'à la jalousie mutuelle du Haut et du Bas-Canada;

$2^{\circ}$ - “... Such union would encourage the introduction of a numerous population from the parent state, and give to the Province of Lower Canada a British Character »;

$3^{\circ}-$ L'union renforcerait le pays contre l'ennemi éventuel;

$4^{\circ}$ - “. . . by encouraging the settlement of waste lands ... such union would greatly augment our commercial resources, increase the value of all real property, and give an impetus to the prosperity and improvement of both provinces, which would render them one of the most valuable appendages of the British Empire."27

\section{$11^{\circ}$ - Adresse des Constitutionnalistes de Montréal aux citoyens d'origine anglaise ou irlandaise (1834).}

Le 16e paragraphe, rappelant les récriminations des Français, confesse que «They (les Français) have denounced, as a mischievous monopoly, a Land Company (la British American, établie à Sherbrooke en 1833) established for the purpose of settling with a British population, lands which, by their distance from a market and want of roads, would otherwise be inaccessible to individual enterprise; wilfully overlooking the fact, that the immense tract of land still held by the Crown, and offered for sale in small parcels, at low rates, secure the advantages of competition, and will prevent the Company from using the privileges to the detriment of the community. $\gg 28$

A la page 396 , le mémoire avoue aussi que « The irresponsible manner in which the Land Granting Department is conducted... Demand revision.»

\section{La résultante}

Ces séries de faits et de témoignages révèlent, il nous semble, un effort réel et constant vers un but précis. Pris à part, un seul de ces faits ou témoignages ne prouverait peut-être pas grand'chose; mais leur multiplicité, leur succession et leur en-

27 Ibid., 330.

28 Ibid., 392. 
chaînement mènent à une déduction inéluctable: les Eastern Townships étaient destinés à une britannisation intense, dont on escomptait, grâce à l'immigration, à l'accaparement des terres, à l'accroissement de la représentation parlementaire, au débordement du peuplement en direction des seigneuries, à l'union des deux Canadas, l'infaillible balayage ou l'absorption de toute nationalité autre que la saxonne.

La lutte menée dans ce sens a eu des protagonistes fanatiques, tels Black, Ryland, Craig, Sewell; mais en général, les meneurs, de Shore Milnes à Durham et à Galt, y vont avec un naturel et une sincérité qui laisse deviner moins de haine que d'assurance: il s'agit simplement pour eux d'établir l'unité politique et nationale.

Ces aspirations un peu simplistes de conquérants ne doivent pas tellement nous étonner, l'histoire confirme qu'elles sourdent comme naturellement après la prise ou l'annexion de maints autres pays: l'Ecosse, le pays de Galles, l'Irlande, la Bretagne, le Congo, l'Afrique, le Pakistan, l'Algérie, la Pologne, l'Autriche et les anciennes principautés d'Italie ont connu ou connaissent les mêmes tentatives de la part de leurs dominateurs.

Ce qui stupéfie et amuse l'observateur, c'est l'ampleur de l'insuccès d'une tactique concertée chez nous avec tant de précaution, d'habileté, de puissance et de ténacité. Comment expliquer que des diplomates soi-disant clairvoyants, que des politiques à qui ne manquaient ni l'expérience ni la perspicacité, se soient si pitoyablement trompés dans leurs prévisions ? D'abord ouverte par des Loyalistes enthousiastes, la région des Townships s'est muée en une tête de pont formellement affectée à l'anglicisation de la province, mais retour bizarre des choses, elle est devenue contre toute attente l'habitat d'une génération française et catholique. En se recoupant, les trois lignes de force, déroutant les calculs ordinaires de la mécanique, ont laissé la plus faible déterminer la résultante.

Les premiers colons ont depuis longtemps perdu leur entité de Loyalistes. Sans doute on retrace leurs noms dans quelquesuns des townships où ils se sont maintenus (Sutton, Brome, Mis- 
sisquoi, Knowlton, Hatley, Stanstead, Eaton, Shipton et Kingsey), mais leur descendance décroissante s'est intégrée dans la pratique à celle des Britanniques: ils n'ont plus de prétentions que celle de préserver du délabrement leurs temples de moins en moins fréquentés, où s'accuse toujours l'émiettement de leurs croyances.

Les Britanniques comptent pour environ $1 / 20$ de la population. Ils détiennent encore le commerce et l'industrie, et la toponymie rappelle obstinément leur antique envoûtement d'impérialistes. ${ }^{29}$ Nombre d'entre eux s'excusent de ne pouvoir s'exprimer en un français coulant, et leurs rencontres avec les Canadiens français s'imprègnent d'une désarmante cordialité. Au parlement, ils n'ont plus guère de représentants de leur race. Et sur le plan religieux, après avoir applaudi à la visite de l'archevêque anglican Fisher, de Westminster, au Souverain Pontife Jean XXIII, ils paraissent prêts à concéder qu'en fin de compte, le Romish Church n'est pas si diabolique qu'on l'a crue aux siècles derniers.

Les Canadiens français se sont mis à pénétrer pour de bon dans les Eastern Townships après 1850. En 1840, l'élément français à Sherbrooke est encore négligeable. En 1851, on y recense 400 Canadiens français, et 1,419 en 1861; en 1871, l'élément francophone et l'élément anglophone s'équivalent en nombre; en 1901, 7,000 noms français sur 11,452 âmes, soit plus de $66 \%$; en $1931,22,000$ sur 28,777 , soit $76 \%$. En 1961, Sherbrooke a 62,000 habitants canadiens-français dans la proportion de $90 \%$ et catholiques dans la proportion de $93 \%$. Notre cité est aujourd'hui plus française que Montréal et que Québec. Les chiffres prennent encore plus d'éloquence si l'on embrasse tout ce qui est historiquement Townships, c'est-à-dire tout ce qui, avant 1854, n'était pas seigneuries: on recense aujourd'hui dans ce même domaine, qui veut s'appeler l'Estrie, une population de 800,000 en chiffre rond, canadienne-française à $95 \%$ et catholique à $96 \%$.

\footnotetext{
29 Dans la seule ville de Sherbrooke, les appellations de pas moins de 40 des anciennes rues font allusion à quelque propriété, haut fait ou personnage rattaché à la cour de Londres.
} 
Les Loyalistes, une fois dominés par les Britanniques, n'avaient guère de chance de reprendre le dessus: accueillis froidement dès le début, gardés ensuite à vue par les gouvernants, ils devaient fatalement, à cause de la similitude de langue sinon de religion, finir par se fondre dans le flot britannique.

Les Britanniques, eux, avaient tous les atouts en mains : avec la richesse matérielle, l'influence politique et le capital humain dont ils disposaient au siècle dernier, il leur eût été facile d'étouffer la nationalité rivale. Peut-être ont-ils trop misé sur ces facteurs caducs, et trop négligé les valeurs spirituelles, qui a la longue s'avèrent les plus vitales pour une civilisation: des peuples pauvres mais croyants comme ceux de la Pologne, de l'Irlande et de la renaissante Acadie pourraient leur donner des leçons sur ce chapitre. L'élément britannique a surtout oublié de se perpétuer, pendant que son émule, la nation française, façonnait des berceaux à la douzaine. Le projet de domination des britannisants sur tout le sud du Saint-Laurent reflétait beaucoup d'ingéniosité, mais il y manquait un proviso essentiel, celui de la postérité. Leur désenchantement éclate aujourd'hui dans l'exclamation désolée d'un de leurs conférenciers: «We are disappearing through our own fault. ${ }^{30}$

Les Canadiens français ne cachent pas leur satisfaction de posséder aujourd'hui le nombre, avec toutes les facilités de développements matériels, religieux et culturels. Le dommage, c'est qu'ils s'exposent à dormir sur leurs lauriers, sans soupçonner que des revirements peuvent encore se produire. Insensiblement, ils subissent à leur tour la sournoise infiltration du «way of life» dont les protège mal la frontière toute proche. L'issue pourrait alors être une espèce de revanche de l'élément loyaliste, présumé mort, mais qui ressusciterait avec l'envahissement de la civilisation américaine. Souhaitons que leur patriotisme les éveille avant qu'ils soient gangrénés dans leur langage, leurs coutumes et leur philosophie de la vie par les importations quotidiennes des mœurs de New-York et de Boston.

MaURICE O’Bready, P.D.

30 Horace Taylor, Knowlton. 